\title{
The changing accretion states of the intermediate polar MU Camelopardalis ${ }^{\star}$
}

\author{
A. Staude, A. D. Schwope, R. Schwarz, J. Vogel, M. Krumpe, and A. Nebot Gomez-Moran \\ Astrophysikalisches Institut Potsdam, An der Sternwarte 16, 14482 Postdam, Germany \\ e-mail: [astaude; aschwope] @aip.de \\ Received 22 December 2006 / Accepted 22 April 2008

\section{ABSTRACT} \begin{abstract}
determine the accretion modes and the accretion geometry from multi-wavelength, multi-epoch observational data.
Methods. Light curves in different observed energy ranges (optical, UV, X-ray) are extracted. The timescales of variability in these detected periodicities and each fitted with an identical model, to quantify the differences in the fitted components.

Results. The published tentative value for the spin period is unambiguously identified with the rotation period of the white dwarf. We detect a distinct soft X-ray component that can be reproduced well by a black body. The analysis of data obtained at different epochs demonstrates that the system is changing its accretion geometry from disk-dominated to a combination of disk- plus streamdominated, accompanied with a significant change in brightness at optical wavelengths.
\end{abstract} \\ Aims. We study the timing and spectral properties of the intermediate polar MU Camelopardalis (1RXS J062518.2+733433) to \\ light curves are determined using Analysis of Variance. Phase-resolved X-ray spectra are created with respect to the most prominent
}

Key words. novae, cataclysmic variables - accretion, accretion disks - X-rays: binaries stars: individual: MU Cam (1RXS J062518.2+733433)

\section{Introduction}

Intermediate Polars (IPs) are close binaries that consist of a magnetic white dwarf and a Roche lobe-filling late-type mainsequence star. Mass loss from the secondary star occurs via the inner Lagrangian point, and the mass is accreted by the white dwarf. In contrast to the true Polars, the white-dwarf rotation is unsynchronised with the orbital motion but is more rapid due to the angular momentum gained from the accreted matter.

In the case of a high accretion rate or a weak magnetic field, the magnetosphere will be smaller than the circularisation radius and an accretion disk will form. The inner disk is truncated by the magnetic field of the white dwarf, which channels the matter via accretion curtains to the magnetic poles. Accretion arcs are formed along the footpoints of accreting field lines. Along these arcs, the accretion rate is assumed to vary. The heated plasma in the accretion zone is a prominent site of hard X-ray radiation, which due to the lighthouse effect is modulated on the spin period, $\omega$. Because of irradiation, the inner-disk rim may emit radiation that is modulated on this period.

For stronger magnetic fields, some or all of the material from the infalling stream might be directly captured by the field without going through an intermediary accretion disk. In the case of this stream-fed accretion, material transferred to the white dwarf retains the orbital motion of the secondary star, producing periodic variability of the beat frequency $\omega-\Omega$, or other sidebands with the orbital frequency $\Omega$.

Most IPs accrete predominantly by means of an accretion disk, V2400 Oph being a rare example of a system with pure stream-fed accretion (Hellier \& Beardmore 2002). A number of

\footnotetext{
* Based on observations obtained with XMM-Newton, an ESA science mission with instruments and contributions directly funded by ESA Member States and NASA.
}

IPs show evidence for accretion by means of both mechanisms simultaneously: via accretion curtains beginning at the inner disk rim, and via so-called disk-overflow when a part of the ballistic stream bypasses the accretion disk outside the orbital plane. In some systems repeated changes between the different accretion states were observed (TX Col, Norton et al. 1997; FO Aqr, Evans et al. 2004). Principal evidence for stream-overflow in IPs has been based on indirect means, i.e. the detection of certain sideband frequencies in their power spectra, which is not always unambiguous. For instance, sideband frequencies may also be produced by spin-pulsed light reprocessed in orbitally-fixed structures (a problem mainly in the optical and UV); alternatively, amplitude modulation of the orbital period (e.g. due to X-ray absorption) may introduce spurious side-bands (Warner 1986).

An article by Patterson (1994) introduces IPs in general, whereas Hellier (2002) discusses the mechanisms of stream-fed accretion.

The X-ray source MU Camelopardalis (the new name according to Kazarovets et al. 2006), for the source formerly known as 1RXSJ062518.2+733433) was identified as an intermediate polar by Araujo-Betancor et al. (2003) and Staude et al. (2003). Optical photometry showed strong variability on mainly two timescales, which were tentatively identified with the orbital period of the system and the spin period of the white dwarf, $P_{\text {orb }}=16987(23) \mathrm{s}=0.19661(27) \mathrm{d}$ and $P_{\text {spin }}=1187.246(4) \mathrm{s}=0.01374127(5) \mathrm{d}$, respectively (Staude et al. 2003). It remained an open question whether the measured spin period provided the white-dwarf rotation, if it was twice the true rotation value, or if it was a side-band period with the orbital variation.

MU Cam is one of a handful of IPs that show a prominent soft X-ray component additional to the hard component 
Table 1. The observations analyzed in this work.

\begin{tabular}{llr}
\hline \hline Date $(\mathrm{dd} / \mathrm{mm} /$ yyyy $)$ & Telescope/Instrument & Duration \\
\hline $28 / 03 / 2005$ & AIP 70 cm/R & $5.0 \mathrm{~h}$ \\
$30 / 03 / 2005$ & AIP $70 \mathrm{~cm} / \mathrm{R}$ & $8.6 \mathrm{~h}$ \\
$20 / 04 / 2005$ & AIP $70 \mathrm{~cm} / \mathrm{R}$ & $4.7 \mathrm{~h}$ \\
$31 / 05 / 2005$ & AIP 70 cm/R & $7.6 \mathrm{~h}$ \\
$31 / 05 / 2005$ & XMM / PN+MOS+OM & $12.5 \mathrm{~h}$ \\
$06 / 04 / 2006$ & XMM / PN+MOS+OM & $10.6 \mathrm{~h}$ \\
$08 / 05 / 2006$ & AIP 70 cm/R & $4.4 \mathrm{~h}$ \\
\hline
\end{tabular}

(Staude et al. 2003). An XMM-Newton observation was proposed to identify undoubtedly the spin period of the white dwarf, and to determine the origin of the soft X-ray radiation.

We report on X-ray and UV observations of MU Cam with XMM-Newton on both March 31, 2005, and April 6, 2006, and coordinated ground-based optical photometry.

\section{Observations}

A short summary of all observations analyzed in this work is provided in Table 1 .

\subsection{Data from $X M M-N e w t o n$}

The X-ray counting EPIC instruments were operated in smallwindow mode with the thin filter. The EPIC-RGS devices were used in the standard configuration. The optical monitor (OM) was kept in fast mode with filter $U V M 2$.

On March 31, 2005 (revolution 972), an observation with XMM-Newton (observation ID: 0207160101), of a scheduled duration of $49 \mathrm{ks}$, was performed. Due to the high background, both EPIC-MOS detectors were switched off after $13.6 \mathrm{ks}$, while the EPIC-PN observed for $30 \mathrm{ks}$. The OM was in operation for $45 \mathrm{ks}$. Ground-based observations were performed synchronously in the $R$-band. The X-ray observations corresponded to 1.75 binary orbits and 25 white-dwarf spin cycles, in which the EPIC-PN collected $\sim 37000$ source counts. A preliminary analysis was presented by Staude et al. (2006). The observed mean source count rates were $1.54 \mathrm{~s}^{-1}$ in the PN, $0.38 \mathrm{~s}^{-1}$ and $0.43 \mathrm{~s}^{-1}$, in the MOS1 and MOS2, respectively; $2.3 \mathrm{~s}^{-1}$ in the OM/UVM2; and $0.031 \mathrm{~s}^{-1}$ and $0.040 \mathrm{~s}^{-1}$, in the first order of RGS1 and RGS2, respectively.

In revolution 1158 (on April 6, 2006, observation ID: 0306550101), XMM-Newton observed the target again for a scheduled $\sim 39 \mathrm{ks}$. All instruments were used for the complete observation duration. Due to a telemetry gap, half an hour of data is missing for all instruments. Additionally, two blocks of $4400 \mathrm{~s}$ exposure in the OM were also lost. During this observation, the mean source count rates were measured to be $1.17 \mathrm{~s}^{-1}$ in the PN; $0.30 \mathrm{~s}^{-1}$ and $0.34 \mathrm{~s}^{-1}$ in MOS1 and MOS2, respectively, $4.2 \mathrm{~s}^{-1}$ in the OM/UVM2; and $0.022 \mathrm{~s}^{-1}$ and $0.029 \mathrm{~s}^{-1}$ in the first order of RGS1 and RGS2, respectively.

The data reduction was completed using XMM-SAS version 6.5; in particular the tasks EPCHAIN, EMCHAIN, and RGSPROC were adopted to process the X-ray data. Source and background photons were extracted in adjoining circles for the PN and for the MOS data from 2006, while the background in the MOS observation of 2005 was extracted from an annulus around the source circle. Events with detection patterns of up to quadruples were selected.

Background-subtracted light curves in different X-ray energy bands - created with EVSELECT - of the PN data are shown in Figs. 1 and 2 with the OM UV-data and groundbased $R$-band data acquired contemporaneously. The energy bands were selected after inspection of the shape of the averaged spectra (Fig. 4), which showed a soft component below $0.7 \mathrm{keV}$, and a minimum in the harder energy range between 2 and $3 \mathrm{keV}$. We therefore define the following PN energy bands: $S X: 0.3-0.7 \mathrm{keV}, M X: 0.7-2.0 \mathrm{keV}, H X: 2.0-12.0 \mathrm{keV}, M H X$ : $0.7-12.0 \mathrm{keV}$, and $T X: 0.3-12.0 \mathrm{keV}$.

EPIC source spectra were created with the task ESPECGET. RMF files for the data from the RGSs were created with RGSRMFGEN. In further analysis, all spectra were grouped with GRPPHA to contain at least 20 counts per bin.

The OM light curves were extracted using the task OMFCHAIN.

\subsection{Optical photometry}

Photometric observations in the optical wavelength range were performed with the $70 \mathrm{~cm}$ telescope of the AIP in Potsdam. Differential photometry for this data was completed using DoPhot. The apparent $R$-band magnitudes were calculated from the brightness of the comparison star as in Staude et al. (2003).

Simultaneously to the observations with XMM-Newton on March 31, 2005, 7.6 h of optical data were acquired with exposure times of $60 \mathrm{~s}$ (see Fig. 1). For the period analysis, further data taken on March 28 and 30, and on April 20, 2005 were included.

Compared to the $R$-band data presented by Staude et al. (2003), the object was almost one magnitude fainter in 2005. In 2003, the optical short-term variability occurred on the period that we would now identify with the true white-dwarf spin (see Sect. 4), whereas in 2005 it occurred on $\omega-\Omega$.

There is no optical photometry simultaneous to the XMM-Newton observation of 2006. Nevertheless, the star was observed for $4.4 \mathrm{~h}$ on May 08, 2006, using the AIP $70 \mathrm{~cm}-$ telescope with $60 \mathrm{~s}$ exposure time (see Fig. 3).

As found for data acquired in 2003, the system was measured to be one magnitude brighter than in 2005 and its variability to be dominated by the white-dwarf spin period (see Sect. 4).

\section{Analysis of the averaged X-ray spectra}

The EPIC-PN and MOS1/2 spectra for both observations are shown in Fig. 4. Both spectra appear similar and have flux in all energy bins in the interval from 0.3 to $11 \mathrm{keV}$. In the energy range below $0.6 \mathrm{keV}$, a distinct soft component is observed, which confirms the classification of MU Cam as a soft IP. Around $6.5 \mathrm{keV}$, contributions from Fe emission lines are detected. The spectral analysis of our data was performed with Xspec 11.3.0.

Staude et al. (2006) fitted the spectrum from 2005 with a model consisting of a hot emitting gas (mekal), a black body component (bbody), and a Gaussian representing iron-K emission lines (Gaussian) absorbed by a photoelectric absorber (wabs), and an additional hot-gas model absorbed by a photoelectric absorber of different column density, i.e. $w a b s_{1}$ $\left(\right.$ mekal $\left._{1}\right)+$ wabs $_{2}\left(\right.$ mekal $_{2}+$ bbody + Gaussian $)$. Since the hydrogen column density, $n_{\mathrm{H}}$, of $w a b s_{1}$ was 250 times higher than that of wabs $_{2}\left(n_{\mathrm{H}}=2.7 \times 10^{22} \mathrm{~cm}^{-2}\right)$, the model consisted of components absorbed by a hydrogen column comparable to the interstellar absorption, and an additional highly-absorbed mekal.

The data from 2005 and 2006 were fitted in the range from 0.3 to $11 \mathrm{keV}$ with Xspec using an identical multicomponent model for both data sets. This model consisted of 


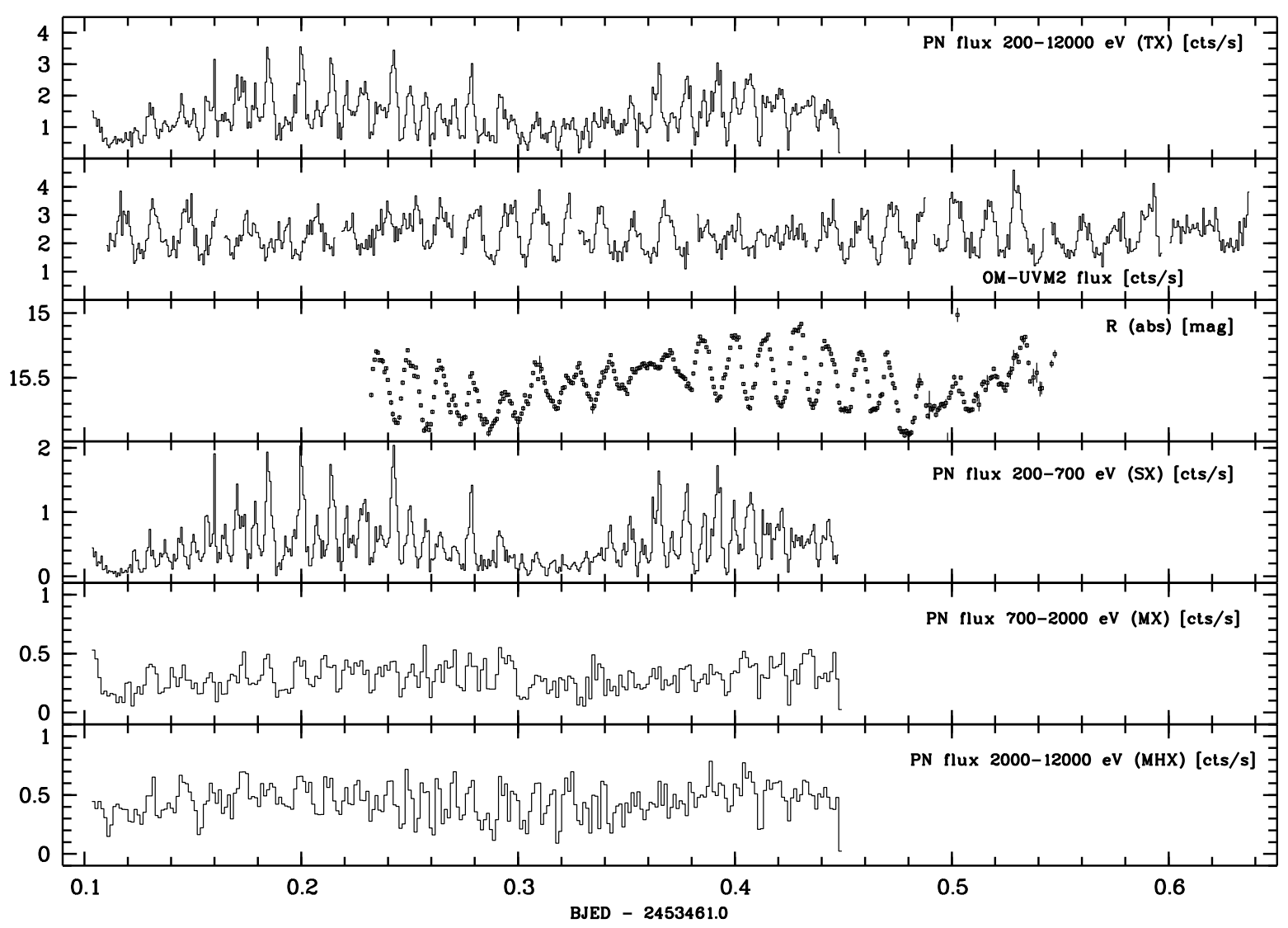

Fig. 1. The light curves of MU Cam, obtained simultaneously on March 31, 2005, by XMM (PN and OM) and with the AIP $70 \mathrm{~cm}$-telescope (R). The data shown here are binned in time, $60 \mathrm{~s}$ for the first, second, and forth panel (counted from top to bottom), $120 \mathrm{~s}$ for the lower two. The $R$-band data are unbinned.

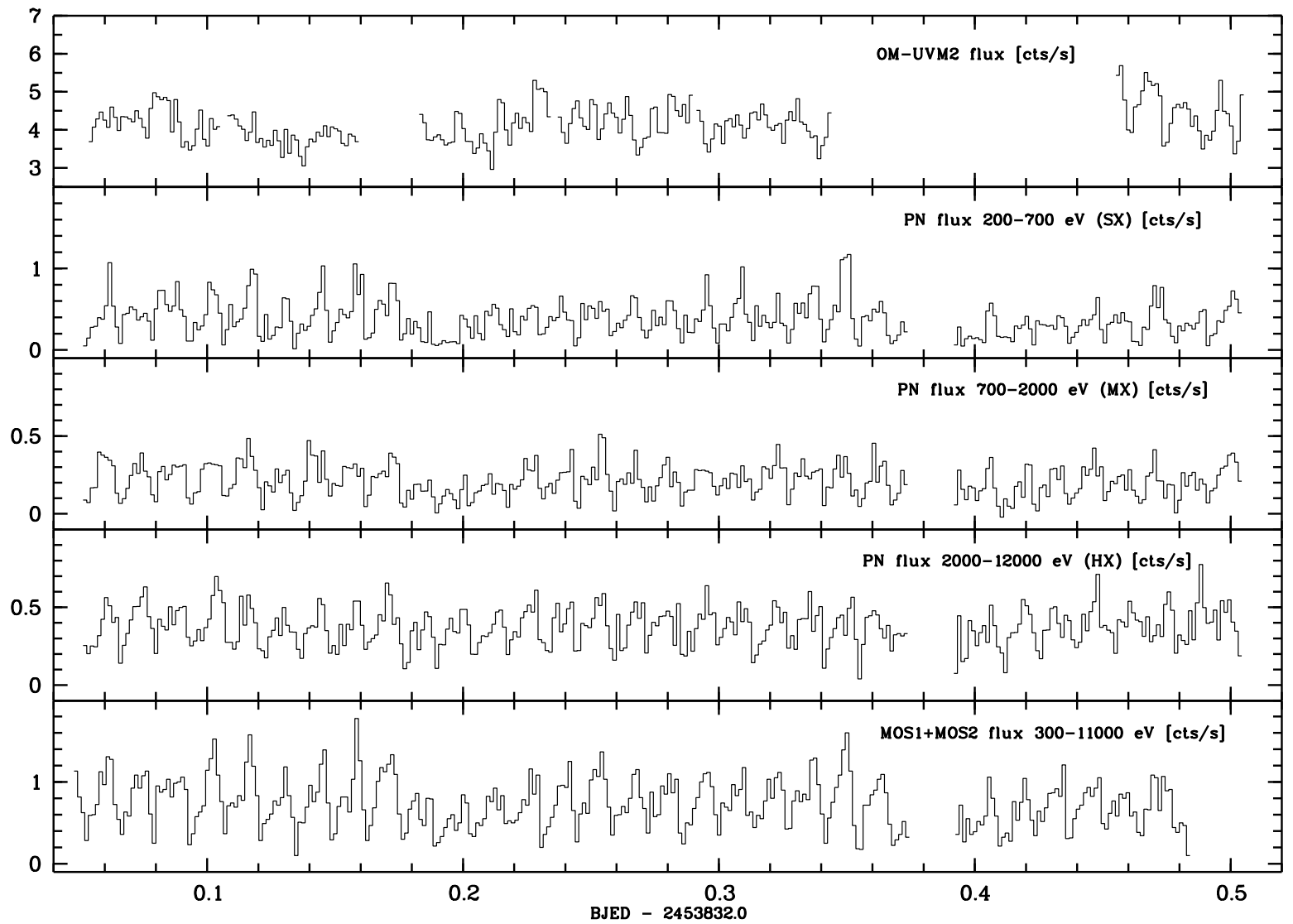

Fig. 2. The light curves of MU Cam, obtained on April 6, 2006, by XMM (PN, MOS1, MOS2, and OM). The data shown here are binned to 120 s. 

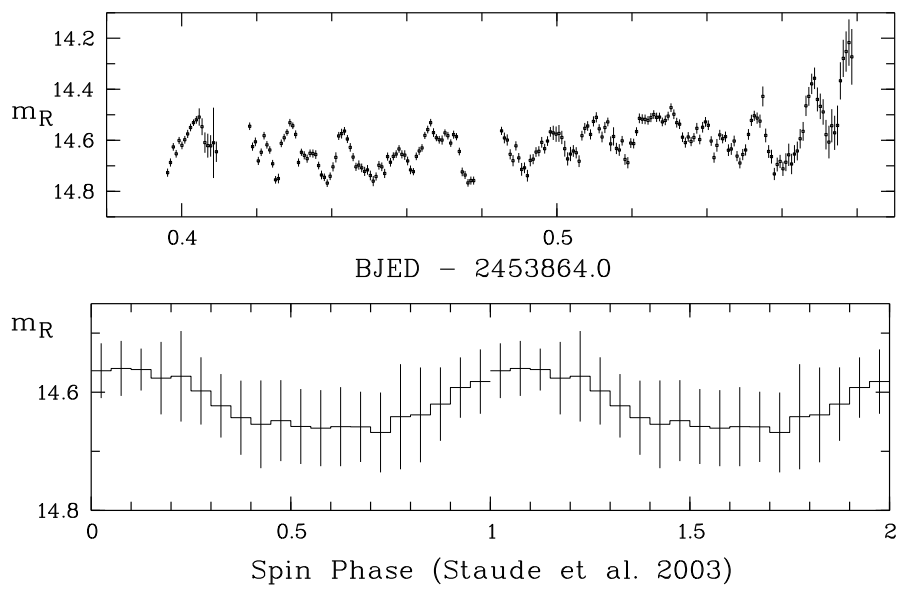

Fig. 3. Top: $R$-band light curve from May 8, 2006, bottom: the above data with BJED $\leq 2453864.56$, binned into 20 bins on the spin period calculated with the ephemeris given by Staude et al. (2003). The error bars mark the standard deviation of the values entering each bin.

an emitting hot-gas component (mekal), a black body (bbody), and two Gaussian emission lines representing the iron-K lines at $6.4 \mathrm{keV}$ and $6.7 \mathrm{keV}$ (Gaussian), which were all absorbed by a partially-covering photoelectric absorber ( $p c f a b s$ ), and a simple absorber representing the interstellar medium (wabs), i.e. $w a b s(p c f a b s($ mekal + bbody + Gaussian + Gaussian $))$. The model fitted the data as well as the previous model, although it required just one partially-absorbed hot-gas component, instead of two of different homogeneous absorption. The model can be understood according to the standard picture of IPs: the bbody represents the soft X-ray emission below $0.7 \mathrm{keV}$, either thermal emission or reprocessed hard X-rays, the mekal component describes the hard component emitted by the hot post-shock material above the white dwarf's surface, and the Gaussian emission line at $6.4 \mathrm{keV}$ (the $\mathrm{Fe}$ fluorescence line) as a tracer of reprocessed X-rays. The pcfabs models absorption inside the system by the accretion curtain/stream. It is mainly responsible for the absorption of soft photons in the mekal component, which explains the relative weakness of flux in the range between 0.7 and $7 \mathrm{keV}$ with respect to flux at the harder end of the spectrum.

Values of the free-fit parameters were determined by $\chi^{2}$-minimisation and are listed in Table 2. The fits were successful in both cases with a reduced $\chi_{v}^{2} \sim 1$. Adding further components such as another mekal or a black body, improved the fit only marginally. A third Gaussian was not required to model the $\mathrm{Fe}$-line at $6.96 \mathrm{keV}$, because this line was described well already by the mekal model. Our fit was not influenced significantly by allowing the metal abundance of the mekal to vary as a free parameter; in this case, the mekal, in fact, hardly changed from its initial value. The lines of iron were resolved in data acquired in 2006 and their widths were therefore allowed to vary, whereas they were unresolved in the 2005 data (see insets in Fig. 4). In the data from 2005, there is line emission at the positions of all three iron-K lines, although an insufficient number of counts do not enable the lines to be resolved. We therefore fixed their line widths to $0.1 \mathrm{keV}$ in the fitting, assuming that they were as broad as in 2006.

The parameters of the partial absorber in our fits are comparable to the values obtained for the other soft IPs observed with XMM-Newton, V405 Aur $\left(n_{\mathrm{H}}=6.1 \times 10^{22} \mathrm{~cm}^{-2}\right.$, CurFract $=$ 0.52 , Evans \& Hellier 2004), PQ Gem $\left(n_{\mathrm{H}}=11.1 \times 10^{22} \mathrm{~cm}^{-2}\right.$, CurFract $=0.45$, Evans et al. 2006), UU Col $\left(n_{\mathrm{H}}=10 \times\right.$ $10^{22} \mathrm{~cm}^{-2}$, CurFract $=0.51$, de Martino et al. 2006), and
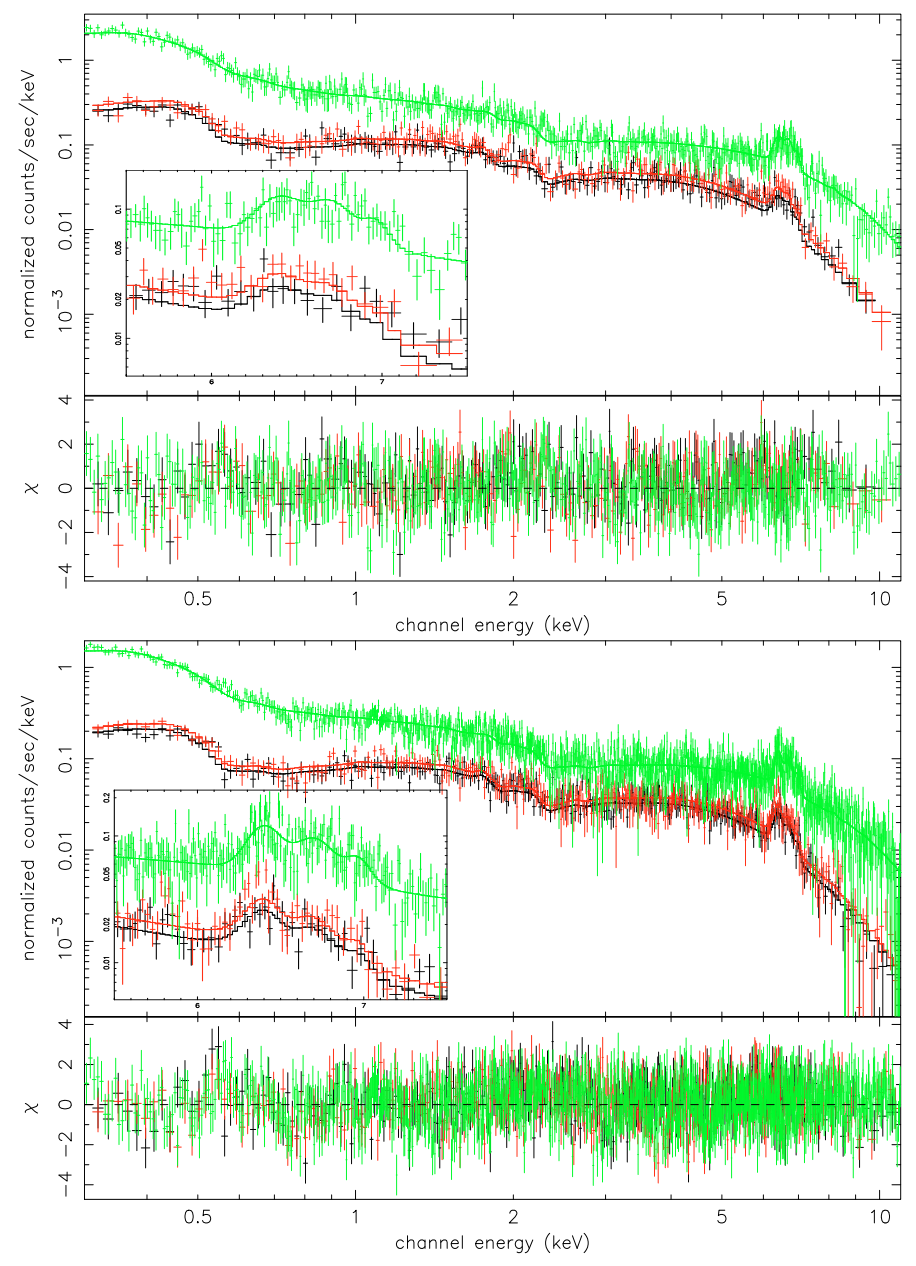

Fig. 4. The EPIC X-ray spectra from 2005 (top) and 2006 (bottom) (green PN, red+black: MOS1/2) with overplotted fit and residuals (Parameters in Table 2). The insets show an enlarged view of the iron-K region.

NY Lup $\left(n_{\mathrm{H}}=9.7 \times 10^{22} \mathrm{~cm}^{-2}\right.$, CvrFract $=0.47$, Haberl et al. 2002).

A problem, however, with our fits is that the fitted temperatures from the mekal models are at the upper limit for the model available in Xspec. When limiting the temperature to lower values, the $N_{\mathrm{H}}$ of the $p c f a b s$ is reduced while its covering fraction is increased, accompanied by a larger $\chi_{v}^{2}$ value of the fit. Haberl et al. (2002) report that fitting a multi-temperature distribution of mekal models with temperatures following a power law to the data of NY Lup, leads to an unconstrained maximum temperature, i.e. a similar problem.

MU Cam was detected to be an INTEGRAL/IBIS source. Barlow et al. (2006) measured a bremsstrahlung temperature of 8.1(4.7) keV. Judging from the XMM-data, this value is clearly too low; we therefore analysed both data sets together. The INTEGRAL spectrum discussed in Barlow et al. (2006) was kindly provided by the authors and is included in Fig. 5. Fitting both data sets simultaneously with Xspec produced no satisfying results because of the large differences in the number of data points and their errors. However, by fixing the temperature of the mekal-component to a number of different values and fitting the remaining parameters, good fits to the XMM-data $\left(\chi_{v}^{2}<1.1\right)$ and a convincing agreement with the INTEGRAL data points (the model was located within the error-boxes of the three data points) were achieved for $k T=35 \pm 10 \mathrm{keV}$. 
Table 2. The main parameters of the spectral model for the average XMM-Newton spectra from 2005 and 2006. The numbers in brackets are the errors and have to be multiplied by the same power of ten as to that for the parameters. "n.h.p." is the "null hypothesis probability".

\begin{tabular}{llll}
\hline \hline & & 2005 & 2006 \\
\hline wabs & $n \mathrm{H}\left[10^{22} \mathrm{~cm}^{-2}\right]$ & $4.1 \mathrm{E}-02(0.8)$ & $4.7 \mathrm{E}-02(0.6)$ \\
pcfabs & $n \mathrm{H}\left[10^{22} \mathrm{~cm}^{-2}\right]$ & $7.9(0.6)$ & $9.5(0.5)$ \\
& CvrFract & $0.61(0.02)$ & $0.67(0.01)$ \\
mekal & $k T[\mathrm{keV}]$ & $79.9[35]^{1}$ & $79.9[35]^{1}$ \\
& norm & $7.3 \mathrm{E}-03(0.4)$ & $6.5 \mathrm{E}-03(0.3)$ \\
bbody & $k T[\mathrm{keV}]$ & $5.9 \mathrm{E}-02(0.07)$ & $5.4 \mathrm{E}-02(0.05)$ \\
& norm & $1.9 \mathrm{E}-04(0.9)$ & $2.2 \mathrm{E}-04(0.9)$ \\
Gaussian & LineE $[\mathrm{keV}]$ & $6.4\left(-{ }^{2}\right)$ & $6.4\left(-{ }^{2}\right)$ \\
& Sigma $[\mathrm{keV}]$ & $0.1\left(-{ }^{2}\right)$ & $0.07(0.02)$ \\
& norm & $3.1 \mathrm{E}-05(0.7)$ & $2.9 \mathrm{E}-05(0.6)$ \\
Gaussian & LineE $[\mathrm{keV}]$ & $6.7\left(-{ }^{2}\right)$ & $6.7\left(-{ }^{2}\right)$ \\
& Sigma $[\mathrm{keV}]$ & $0.1\left(-{ }^{2}\right)$ & $0.09(0.03)$ \\
& norm & $3.1 \mathrm{E}-05(0.7)$ & $2.5 \mathrm{E}-05(0.7)$ \\
$\chi_{v}^{2}$ (d.o.f) & & $1.034659(948)$ & $1.046327(1754)$ \\
n.h.p. & & $2.2 \mathrm{E}-01$ & $8.7 \mathrm{E}-02$ \\
\hline
\end{tabular}

${ }^{1}$ The temperature of the mekal component ran against its upper limit. The value in brackets is the plasma temperature which is compliant with both the XMM and INTEGRAL spectrum (see Fig. 5 and Chap. 3 ). ${ }^{2}$ The parameters were fixed to these values.

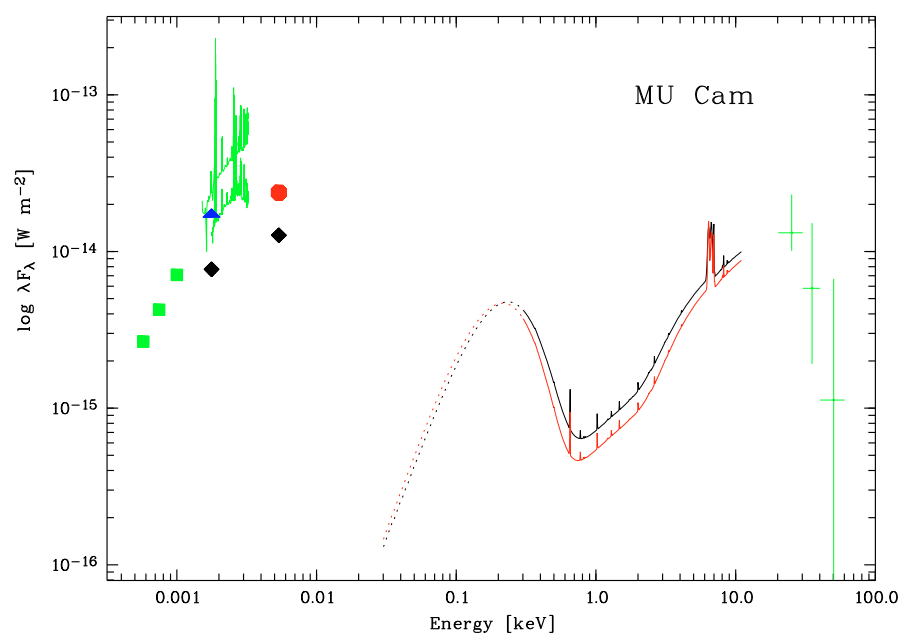

Fig. 5. The spectral energy distribution of MU Cam. Shown are the data synchronously obtained in 2005 (rhombs and X-ray model spectrum in black), the data from 2006 (XMM-Newton: circle and X-ray model spectrum in red, $R$-band flux from the $70 \mathrm{~cm}$ telescope: triangle), and, additionally, 2MASS $J, H$, and $K$ fluxes (squares) plus the optical spectra from Araujo-Betancor et al. (2003) (lower) and Wei et al. (1999) (upper). Additionally, the three data points from the INTEGRAL/IBIS spectrum (Barlow et al. 2006) are plotted with their corresponding error bars. The X-ray models are plotted without the interstellar absorption component. To illustrate the shape of the black body component in the $\mathrm{X}$-rays, the extrapolation to lower energies outside the fitted range is plotted with dots.

Utilizing instruments capable of observing a wider range of X-ray energies (e.g. RXTE), the plasma temperatures in IPs can be determined more precisely (Suleimanov et al. 2005). Compared to the temperatures measured for other IPs, the value of $k T=35 \mathrm{keV}$ is unusually high. Typical values for IPs are $10-20 \mathrm{keV}$. Since this temperature must be regarded as an average of the entire temperature range, and since the reflection component present in the star redistributes photons to higher energies, the post-shock temperature remains only weakly constrained.

The EPIC-spectra and the parameter values of the fits to the two data sets of 2005 and 2006 appear to be quite similar, which is surprising when the differences in the light curves, particularly in the soft X-rays, are considered. The spectra from RGS1 and RGS2 of both epochs were grouped to contain at least 20 counts. The fit to the integrated EPIC spectra reproduces the data well. No significant lines were detected in the wavelength range considered, and we therefore disregard the RGS data in further analysis.

The overall spectral energy distribution of the system is shown in Fig. 5, where the available measurements are plotted with colours that differentiate between data obtained simultaneously (black and red), acquired in an optically-bright state as assessed from the $R$-magnitude (blue), and of unknown state (green).

During an epoch of high optical brightness, the X-ray flux is lower than when the system is optically faint. It is evident that the black body component, which can be seen in the X-rays, contributes only negligibly to the optical/UV flux. The shape of the continuum in the optical/UV wavelength range resembles that of black body with a much lower temperature and can be attributed to the accretion disk.

\section{Variability analysis}

The light curves derived from the observations during the two epochs are shown in Figs. 1 and 2, the corresponding periodograms in Fig. 6. The analysis is supported by additional optical photometric data that are listed in Table 1. In spite of differences between data for different epochs, the periodograms provide a clear measurement of the basic periods of the system.

\subsection{The basic frequencies}

Araujo-Betancor et al. (2003) and Staude et al. (2003) gave a prelimininary identification of the system orbital and spin periods from optical photometric data. Optical spectroscopy was used by Araujo-Betancor et al. (2003) to identify the orbital frequency, $\Omega$. Using the optical data alone, it was impossible to identify unequivocally the white dwarf spin frequency, $\omega$.

Figure 6 shows the results of a period analysis using the method of analysis-of-variance (AOV, Schwarzenberg-Czerny 1989) in the different energy bands, for data from the two XMM campaigns. We indicate the orbital frequency $\Omega$, the tentative spin frequency $\omega$, the side-bands of both these frequencies, and the (sub)harmonics. AOV tends to create artifacts in the form of sub-harmonics; this is in contrast to the Fourier-transform, which creates harmonics. The main peaks in the X-ray bands $H X$ and $M X$ are in both datasets at the assumed $\omega$. In the $S X$ band, the pattern is more complex in 2005 than in 2006, showing variability not only at $\omega$ but also at $\Omega$ and $2 \omega-\Omega$. The OM-data show variability in the so-called beat-frequency $\omega-\Omega$, at least in data from 2005. In the optical $R$-band, the peak is located at either $\omega$ (2003 and 2006) or $\omega-\Omega$ (2005).

A consistent explanation for the presence of all peaks in the periodograms can be given when the tentative value of $\omega$ is assumed to measure the white dwarf spin.

The X-ray periodograms from 2006, which show variability power only at $\Omega, \omega$, and $2 \omega$, could be produced by accretion from a truncated disk to two opposite accretion regions via shock-heated curtains (Wynn \& King 1992); reprocession on the 

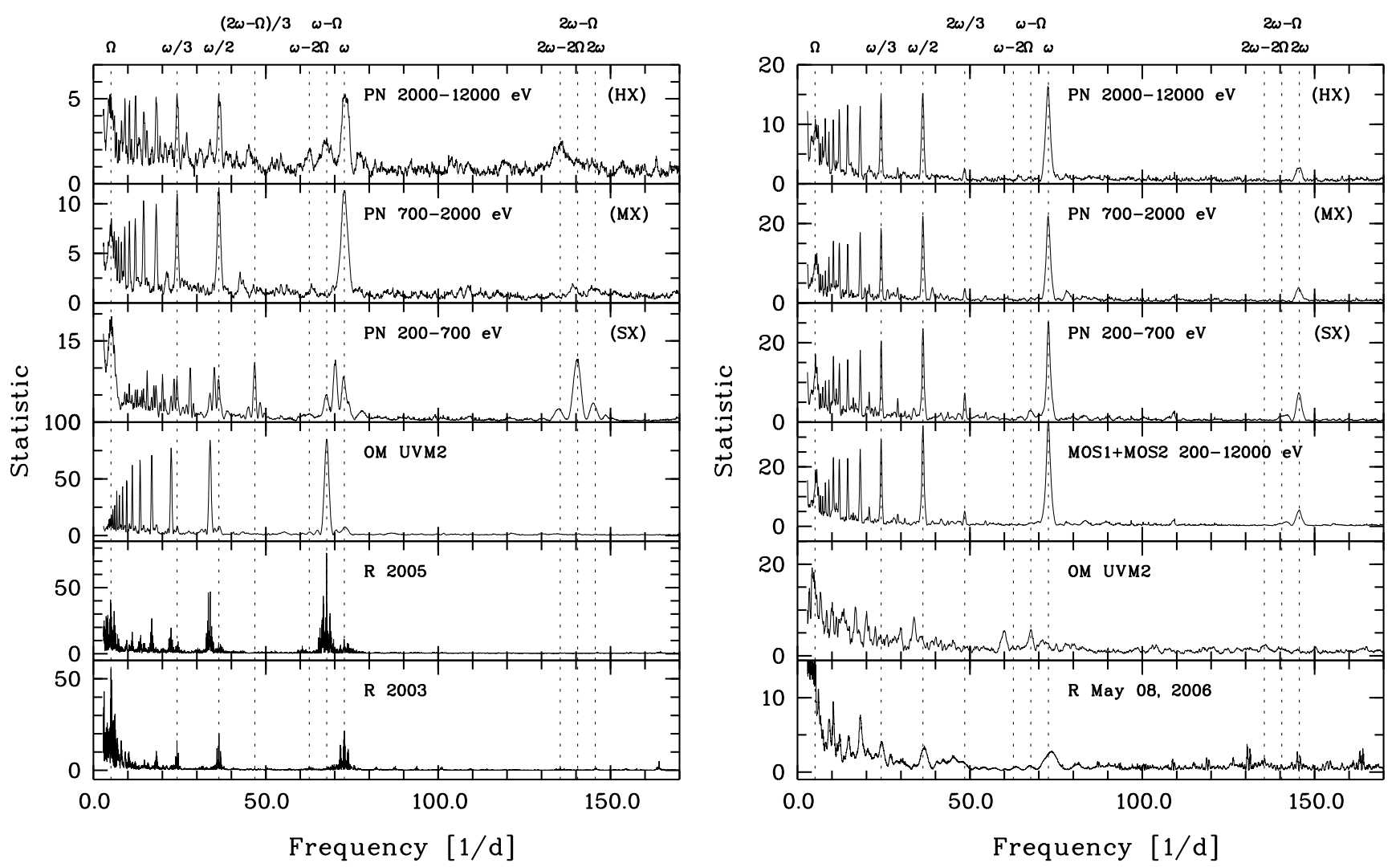

Fig. 6. AOV-periodograms of left: the data from 2005 (Fig. 1) - PN counts in different energy ranges, OM with UVM2, and $R$-band photometry for comparison, the AOV analysis of the $R$-band data from 2003 (Staude et al. 2003) is added; right: the data from 2006 (Fig. 2) in different energy ranges and instruments. The orbital $(\Omega)$ and spin $(\omega)$ frequencies, their sidebands, and (sub-)harmonics are marked with dotted lines.

white dwarf surface would then produce the soft X-rays $(S X)$. In 2005, the harder X-rays $(H X$ and $M X)$ were created by accretion along curtains, indicated by variability in mainly $\Omega$ and $\omega$. The soft X-rays $(S X)$ have variability power also in the frequency $2 \omega-\Omega$ and some signal at $\omega-\Omega$. This can be explained by their origin in reprocession on the white dwarf as well as by accretion directly from the accretion stream via magnetic field lines, which would produce modulations in superpositions of $\Omega$ and $\omega$ (Wynn \& King 1992).

In 2005 , the UV data shows variation mainly at $\omega-\Omega$, the periodogram has weak power only at $\Omega$ and $\omega$. The $R$-band data has a similar variability pattern with stronger variation in $\Omega$. Changes in the dominating frequency from $\omega$ to $\omega-\Omega$, especially at the lower energies, can be described by changing accretion modes within the current models of IPs.

We could imagine assuming the tentative $\omega$ to be the true $\omega-\Omega$. This would be consistent with diskless accretion along magnetic field lines. Certain problems arise in this approach, for example the need to explain the absence of any signature of variation with frequency $\omega$. Since the visibility of the accretion regions is modulated by this frequency, it is expected to be observed in hard X-rays for which absorption is probably incomplete. In the $R$-band and UV data from 2005 , one would have to explain the strong signal at $\omega-2 \Omega$ (the tentative $\omega-\Omega$ ), a small contribution to $\omega-\Omega$, and no trace of $\omega$.

Warner (1986) explained an $\omega-2 \Omega$ varibility to be an orbital modulation of the $\omega-\Omega$ component. It is therefore unlikely that the latter has a far smaller amplitude. We would also have to replace the convenient explanation of $2 \omega-\Omega$ proposed by Wynn $\&$ King (1992), by one for $2 \omega-2 \Omega$; furthermore, for the 2006 data, we would have to consider $2 \omega-\Omega$ without a signature for the $2 \omega$ periodicity.

To summarize, it is unlikely that this second interpretation reproduces our observations of MU Cam. We therefore claim that we have found the spin period predicted by Staude et al. (2003) to be the true rotation of the white dwarf.

Aside from their common properties, the data from the two epochs show a number of differences.

In the light curves from 2005 (Fig. 1), the soft X-rays (SX) and the $R$-band data show a strong dependence of variability amplitude on the orbital phase. In the soft X-ray light curve, two peaks per spin-cycle of various heights are directly visible. They are probably the origin of the frequency $2 \omega-\Omega$.

The most striking differences between the light curves obtained on April 06, 2006 (Fig. 2), and those from 2005 (Fig. 1) is, that there is a reduction of the $S X$ countrate, and the amplitude of the short-term variation does not depend obviously on the orbital period. The orbital period $\Omega$ is detected in the periodograms of all the X-ray bands, although there is no obvious variation of this timescale in the light curves (see Fig. 10).

The $R$-band data - in agreement with the XMM-Newton data, although these were acquired one month later - demonstrate variability mainly with $\omega$. Due to the short duration of the observation - slightly less than one orbit - no secure statement about the variability on the orbital period can be made.

\subsection{Towards a refined spin ephemeris}

Using the data acquired in 2005 and 2006, we attempt to improve the accuracy of the spin ephemeris. The optical data of long-scale base line can be used to refine the period, while the 
hard X-rays relate the ephemeris zero-point to the rotation of the white dwarf.

From the spin-phase folded light curve of the $R$-band data from 2006 (Fig. 3), a new time of spin maximum for a long-term ephemeris can be derived. The phase of maximum, 0.075, corresponds to $T_{\text {max,spin }}(\mathrm{BJED})=2453$ 864.5668(3). The error in the epoch is assumed to be half the bin width, 0.025 spin phases. The cycle-count is lost due to the error in the spin ephemeris from Staude et al. (2003).

Kim et al. (2005) proposed a refined spin ephemeris, $P_{\text {spin }}=$ $0.01374116815(17) \mathrm{d}$, based on a 3-period fit. When folding our $R$-band data from May 8, 2006 with their ephemeris, the spin maximum, however occurs at phase $0.725(25)$, which is clearly outside the error ranges of their ephemeris zero point at the time of observation ( 0.01 phases). The reason for this discrepancy may be that they used data obtained in different accretion states: this is obvious from the significant change in brightness between their measurements in $2004\left(m_{R} \sim 14.5\right)$ and $2005\left(m_{R} \sim 15.5\right)$. We detected fundamental differences in the variability patterns under similar conditions, i.e. a change from variation with frequency $\omega$ to frequency $\omega-\Omega$.

We calculated an X-ray spin-ephemeris from phase-binned light curves of the harder photons (MHX, 700-12000 eV, Fig. 7). Times of maximum flux were detemined from a spline fit to the binned data to be $T_{\max , P N_{\text {hard }}}(\mathrm{BJED})=2453461.4475(8)$ (phase $0.68 \pm 0.02$ ) and $T_{\max , P N_{\text {hard }}}(\mathrm{BJED})=2453832.0615(8)$ (phase $0.57 \pm 0.02$ ). The assumed uncertainty in the maximumdetermination is one bin-width. The flux maxima were used because they represent the time of optimal visibility for the principal accretion region of the white dwarf. Depth and position of the minimum may be strongly dependent on the size of the accretion region(s), because their extension determines the length and completeness of their occultation behind the white-dwarf limb. The apparent optimal visibility of the main accretion region is only weakly dependent on the size.

The spin-ephemeris of Staude et al. (2003) provides a cyclecount of 26971, which corresponds to a period of $P_{\text {spin,MHX }}=$ $0.01374120(6) \mathrm{d}$.

This value matches the previously-published results well. The measurement of the period confirms the previous estimate for an independent data set, although the period does not appear to be more accurate than that derived from optical photometry in 2003. We note, that the spin period measured by Kim et al. (2005), is within the error range of our results.

The timing information derived from optical photometry cannot be combined with that of the X-ray data, because the two types of radiation originate in completely different parts of the binary system, even if their measured variabilities have similar periods: a time-lag between the pulses is therefore expected.

\subsection{From periodicity to structure}

In 2005, the spin-folded light curve in band $M H X$ is sawtoothshaped (Fig. 7), with a slow rise to maximum in $\sim 0.7$ phase units, while the decline lasts only 0.3 phase units. The light curves have a pulsed fraction of 50 per cent and show a similar behaviour in the energy sub-bands $M X$ and $H X$.

In 2006, the $M H X$ light curve looks similar to that from 2005, but its minimum is more pronounced with a pulsed fraction of 75 per cent compared to 50 per cent in 2005.

To obtain more information about the origin of the spectral components, a phase-resolved spectral analysis was performed with respect to different periodicities $(\omega, \Omega, \omega-\Omega, 2 \omega-\Omega)$. The data were binned into 10 phase bins for each period. A number of
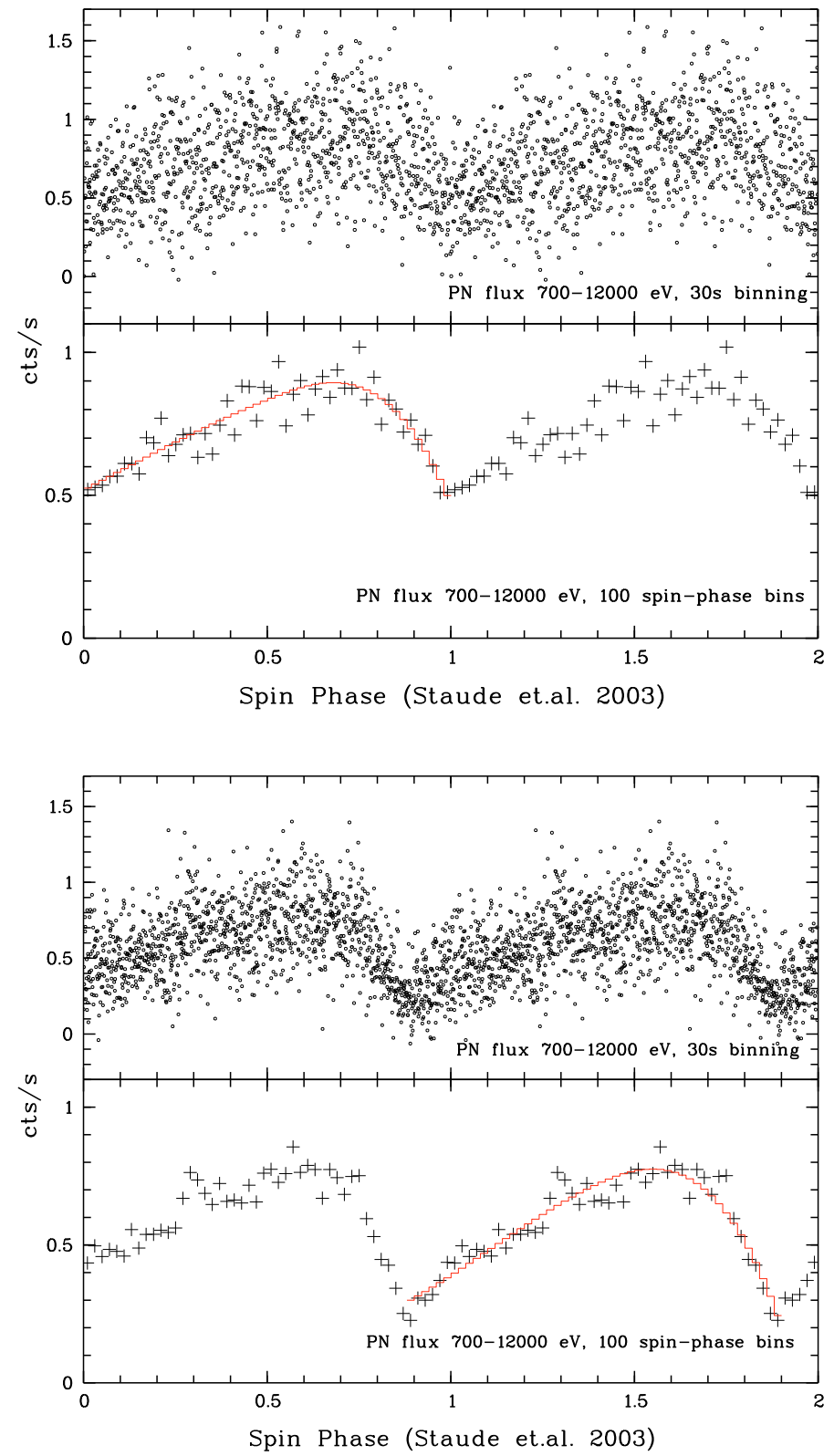

Fig. 7. The background-subtracted PN count rate in the energy band MHX (700 to $12000 \mathrm{eV}$ ) (top 2005/03/31, bottom 2006/04/06) binned to $30 \mathrm{~s}$, plotted over spin-phase (upper). The same data, binned into 50 phase bins with an overplotted spline fit (lower).

parameters from the model fit to the global spectra were fixed to the values previously obtained: $w a b s \_n H$ (since it is interpreted to be of interstellar origin), pcfabs_nH, mekal_kT (because this was, in any case, not constrained), bbody_kT (which when allowed to vary, changed very little around its mean value), and Gaussian(1,2)_sigma.

The error bars in the binned light curve plots indicate the standard deviation of values that enter the bin. We do not calculate the errors in our phase-binned dataset by measuring the Poisson noise of photons in a given bin. This would be the correct approach only when a system was observed repeatedly in a similar state and would show variability only on the period with which the data are folded and binned.

When binning data that are varied by multiple periods, the measurements from different cycles, entering the same bin, are not in general obtained when the system is in a similar state. If 

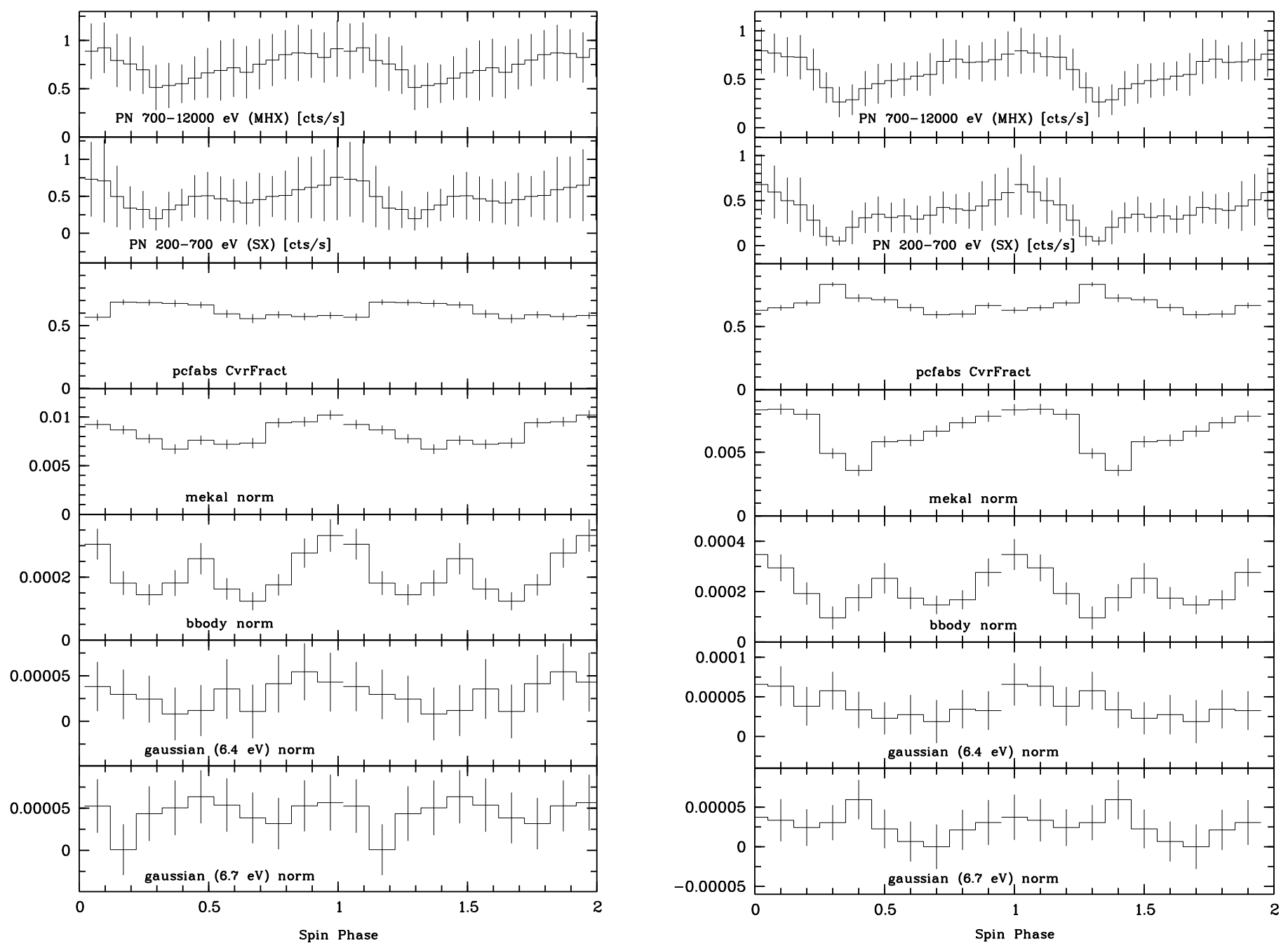

Fig. 8. The spin-folded PN light curves from for 700-12 $000 \mathrm{eV}(M H X)$ and $200-700 \mathrm{eV}(S X)$. For the calculation of the spin-phase, we use as zero points the epochs of maximum flux in the MHX band determined in Sect. 4.2 and the period from Staude et al. (2003). The error bars in the light curves mark the standard deviation of the values having entered the bin. Below are plotted the spin-phase dependent parameter values for the fit to phase-selected MOS+PN spectra. The parameters not shown here were kept constant at the values determined from the fit to the average spectrum. Left: March 31, 2005, right: April 06, 2006.

Poissonan noise was assumed to represent the error, this would not take account of the intrinsic variability by the superposition with the other periods, and would underestimate the errors.

In our data for MU Cam there is always variability on multiple periods - in the simplest case for just $\omega$ and $\Omega$. We could attempt to remove the variation that occurs on one period from the data, but this will certainly not produce the desired result in the presence of strong side-bands, as in the $S X$-band in the 2005 data.

To maintain the size of errors in all plots of binned light curves, we consistently use the standard deviation of values as they enter each bin throughout the paper. These values represent upper limits to the true errors.

\subsubsection{The white dwarf rotation - $\omega$}

The parameter values of the fits to the spin-phase selected spectra are shown in Fig. 8 with phase-folded light curves in the bands $S X$ and $M H X$. The similar shape of the soft phase-folded $\mathrm{X}$-ray light curves is remarkable, despite the obvious differences in the time domain (compare Figs. 1 and 2). It appears that the strong variations in the 2005 data are completely removed when the data are folded and averaged using the spin period.

\subsubsection{The stars' orientation matters $-\omega-\Omega$}

Variability at the beat-period $\omega-\Omega$ was detected only in the data from 2005. It was the dominating period in the OM and the $R$-band light curves, it was clearly present in the soft X-ray band $(S X)$, whereas it was almost undetected in the hard X-ray bands. The beat-phase folded X-ray light curves and phase-resolved spectra do not show significant variation and are therefore not shown.

\subsubsection{A signature of two-pole accretion $-2 \omega-\Omega$}

While the X-ray data from 2006 varies predominantly at the spin period, the soft X-ray component in 2005 shows variation also with additional frequencies, most prominently $2 \omega-\Omega$. This is an indication that there are two accreting poles, whose accretion rates are dependent on the orientation of the white dwarf within the binary frame, i.e. of both stars (Wynn \& King 1992). To decide whether there is any difference between the emission 


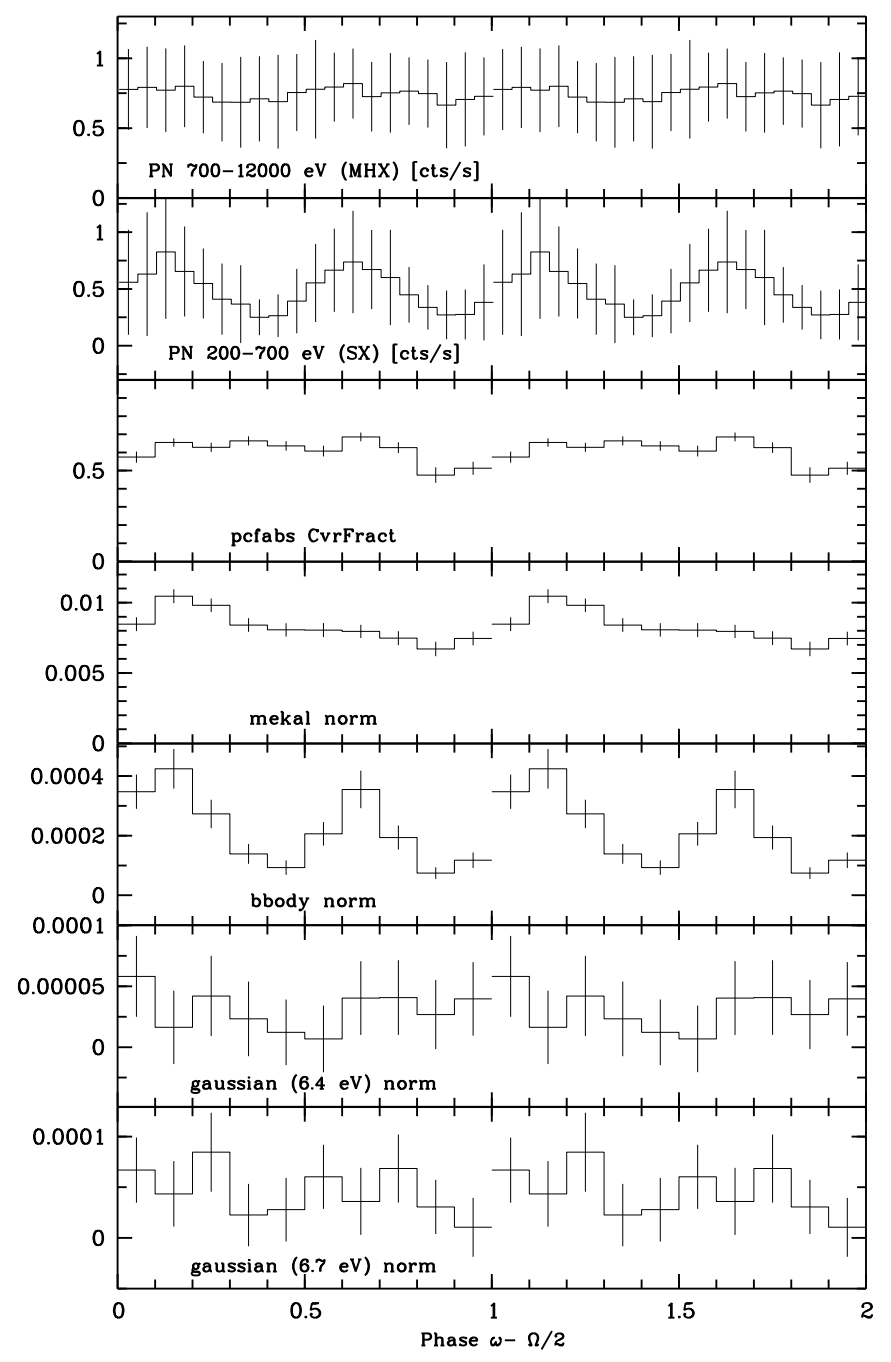

Fig. 9. Light curves in the hard and soft X-ray bands from 2005 folded on the period corresponding to $\omega-\Omega / 2$ (error bars in the light curves are the standard deviation of the values having entered the bin), and the free parameters of the fits to the phase-resolved spectra.

of the two accretion spots, the soft and hard X-ray photons were phase-folded on the period corresponding to $\omega-\Omega / 2$. Spectra were extracted for ten phase-bins with respect to this period $\left(\Phi_{\omega-\Omega / 2}=(B J E D-2452682.4181) / 0.01423886\right)$ and fitted. The light curves and the fitted parameters are shown in Fig. 9.

Separated by 0.5 phases, there are two humps that have similar a soft X-ray light curve, which explains the strong signal of frequency $2 \omega-\Omega$. The parameters of the fit indicate that the source of this variability are two distinct poles: the mekal component has one peak only above an almost constant level, and the integrated black body normalization of the humps clearly differ.

\subsubsection{The orbital period $-\Omega$}

For the data discussed here, a significant and likely periodic dependency of the radiation on the orbital motion is detected only for 2005 (see Fig. 6). Compared to the power in other detected frequencies, the orbital signal is strongest in the soft X-rays, and detected clearly in the other X-ray bands and the optical. Phasefolded light curves and fitted parameter values for the X-ray spectra from 2005 are shown in Fig. 10. There is almost no variation in the UV light curve (which is therefore not shown), but the other light curves display clearly a single hump. The maxima

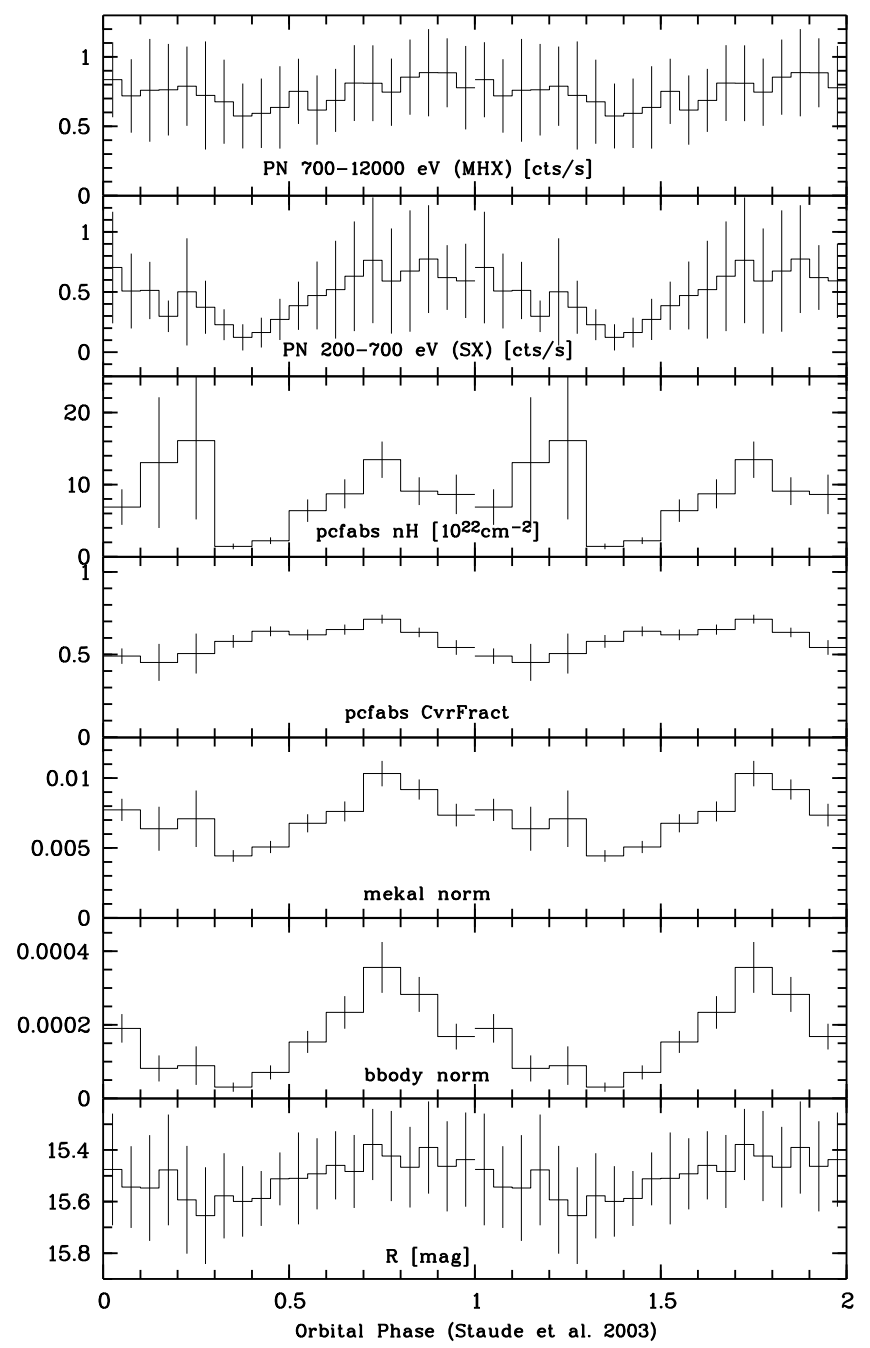

Fig. 10. The orbital period $\Omega$ : light curves from 2005 in the hard and soft $\mathrm{X}$-ray bands, the fitted spectral parameters, and the phase-folded $R$-band data. The error bars of the light curves are the standard deviation of the values having entered the bin.

and minima in the curves do not occur at the same orbital phases - the $R$-band is 0.1 phases ahead. The probable origin of the variability $\Omega$ is the changing visibility of emission sites during the rotation of the binary.

In contrast to the spectral fits applied to data averaged using the other frequencies (see previous sections), the parameter $p c$ fabs_nH was allowed to vary for studying the $\Omega$-dependence of the data from 2005. By fitting the binned data, we searched for variations in the hydrogen column density, caused by the changing orientation of the magnetically-guided stream with respect to the line-of-sight during one revolution. The number of observed orbital cycles, however is rather low for the binned data to be regarded as representative of the mean system; since fewer than two consecutive orbits were observed, the data can be considered only as a snap-shot of the system at a certain time.

The data from 2006, phase-folded on $\Omega$, are not shown, because only marginal variability is observed in the X-ray light curves and OM data; the latter data set and optical data are affected by insufficient coverage of the orbital cycle. We therefore do not attempt any phase-dependent analysis. 


\section{Discussion}

\subsection{Pure disk accretion observed in 2006}

In all of the X-ray periodograms, the dominant frequency is the white-dwarf spin $\omega$. The frequency $2 \omega$ is also present, but far weaker. In the periodograms, there is signal at the orbital frequency $\Omega$ in all wavelength ranges, although it is not prominent in X-rays and not completely resolved in optical light.

Following the analysis of Wynn \& King (1992), the natural explanation for the detected variability pattern is accretion via an accretion disk and accretion curtains, which connect the disk to the magnetic poles. The weak detection of signal at frequency $2 \omega$, in addition to that at $\omega$, implies that there are two accreting spots, almost opposite to each other Their properties or visibility must differ to explain the far stronger signal at $\omega$. This interpretation is supported by Fig. 8 (right panel), where the soft X-ray light curve shows (when compared to the hard profile) a second maximum shortly before phase 0.5 , and the normalisation of the black body component of the phase-resolved spectral fits has a secondary maximum at this position. The normalisation of the mekal-component shows only one maximum - located at the phase of maximum brightness - with a slow rise and a rapid decay. The sawtooth-like shape indicates its origin in an arc-shaped region. The covering-fraction of the absorption component in the spectral fits shows a smooth modulation on the spin period with a clear maximum at the phase of minimum X-ray flux.

The resemblance of the spin-folded X-ray light curves in the two energy ranges to the ones shown by Evans \& Hellier (2004) for V405 Aur is striking. As observed there, the soft X-rays show a double-hump, whereas the hard ones form a sawtooth shape. A similar accretion geometry, therefore, appears to exist in MU Cam, where the white-dwarf dipole axis is located close to the orbital plane instead of being aligned with the spin axis. The hard and soft X-ray light curves and the parameters of the fits to the spin-resolved X-ray spectra (Fig. 8, right panel) can be explained in a model where the lower accreting pole has optimal visibility at spin phase 0.45 , and the upper at phase 0 . Between phase 0 and phase 0.3 , the covering fraction of the partial absorber increases. This absorbing matter is the accretion curtain to the upper pole, crossing the line-of-sight to the X-ray emitting region. The phase-shift between the maximum absorption and the optimal visibility of the accretion region can be explained by the bulk of matter being accreted along trailing field-lines, as proposed by Beardmore et al. (1998) to explain absorption features in observations of FO Aqr. At phase 0.25, the shocked region above the upper pole, which emits hard X-rays, disappears behind the white dwarf, in a similar way to the soft X-ray spot. An indication that soft $\mathrm{X}$-ray emission traces reprocession sites of hard X-rays is the modulation of soft X-rays by the whitedwarf spin.

In contrast to the optical data discussed in Evans \& Hellier (2004), our $R$-band data from 2003 and 2006 do not show a double-peaked spin pulse. The spin-folded optical data from 2003 (see Fig. 4 in Staude et al. 2003) have a zigzag-like shape of amplitude $\sim 0.2 \mathrm{mag}$. The spin-phase folded $R$-band light curve from 2006 (Fig. 3) shows a sinusoidal variation of amplitude $0.1 \mathrm{mag}$. Using the hard X-ray determined spinephemeris (Sect. 4.2), the flux maximum for the 2006 data occurs at phase 0.55 , i.e. half a spin cycle later than the X-ray maximum. We propose that the pulsed fraction (10 percent) of the optical flux originates in the irradiated parts of the accretion curtains. Their irradiated inner regions are most easily visible when the upper pole points away from the observer. The magnetic axis of the white dwarf must be tilted, with respect to the rotational axis, by approximately the system inclination angle to be able to explain the maximum X-ray flux that is half a spin-cycle offset from the optical maximum. When pointing towards the observer at phase zero, this prevents obscuration of the upper accretion region by its accretion curtain.

Unfortunately, the OM light curve shows a clear variation pattern only towards the end of the observation and is affected by data gaps; it is therefore impossible to cross-correlate the OM light curve with the X-ray and optical light curves to measure the relative phasing, i.e. the position of the reprocessing sites.

\subsection{Accretion via disk and stream observed in 2005}

The hard X-ray component is assumed to originate in a shock inside the accretion column above the magnetic pole. Since there is almost no variability in the count-rate of the hard photons on the beat frequency $\omega-\Omega$, the accretion rate does not appear to be dependent on the white-dwarf orientation within the binary frame. The accretion producing the emission of hard photons probably happens via an accretion disk, as occured in 2006.

The shape of the distribution of the soft X-ray photons ( $S X$, $200-700 \mathrm{eV}$ ) is reproduced well by a black body, they appear to originate on the white dwarf surface (because of the strong signal at $\omega$ ). The presence of $\omega-\Omega$ shows that the orientation of the white dwarf in the binary frame has influence on the emission. Stream-fed accretion, penetrating and heating the whitedwarf surface, is probably therefore the origin of (parts of) the soft X-ray component, because pure reprocession of hard X-rays on the white dwarf would produce a signal at $\omega$ only. The frequency $2 \omega-\Omega$ implies that there is a second accreting pole that is opposite to the principal pole, since it denotes the detection of a similar accretion geometry after just a little more than half a spin-cycle, in the case of stream-fed accretion (Wynn \& King 1992). The two peaks per spin cycle can also be directly observed in the light curve (Fig. 1). Figure 9 shows that only the soft X-ray flux (i.e. the flux in the black-body component), not the hard X-ray flux, is modulated with respect to the orientation of the two stars.

As the dominant frequency in the optical and UV light in 2005 , the beat-frequency $\omega-\Omega$ indicates that the emission or visibility of this radiation component was strongly dependent on the orientation of the white-dwarf magnetic field in the binary reference frame. The accretion disk may have been smaller in 2005 than in 2006, and the inner rim therefore located at a greater distance from the white dwarf. This would allow a diskoverflowing part of the ballistic stream, which would otherwise be negligible, to dominate accretion via the disk. Only the prolonged ballistic stream would have a sufficiently high density and incident flux to re-emit significantly, producing a $\omega-\Omega$ periodicity. This view is supported by the lower brightness of the system in the optical/UV in 2005. It is observed that the amplitude of the short-term variations did not vary significantly, in contrast to the size of the constant component.

The orbital variation in the X-ray emission (Fig. 10) is probably caused by the changing visibility of the accretion sites in the binary's frame of reference during an orbital revolution. Our fitted parameters argue against a model, pushed forward by Hellier et al. (1991) to explain orbital modulation of the X-rays in AO Psc, where the minimum at orbital phase 0.3 is explained by increased absorption, e.g. by matter lifted out of the orbital plane. Unfortunately, the published orbital ephemerides either have too large errors (Araujo-Betancor et al. 2003; Staude et al. 2003) or a remaining cycle-count ambiguity (Kim et al. 2005) to allow to relate the relative phasing of the other detected periods 
to the probable epoch of inferior conjunction of the secondary star, as determined by Araujo-Betancor et al. (2003) from phaseresolved spectroscopy.

To explain the behaviour of the system during the XMM-Newton observation in 2005, we therefore propose that the white dwarf was accreting via an accretion disk and a diskoverflowing stream. The hard X-ray emission, which is particularly sensitive to variations on the white-dwarf spin period, are produced by shocks above the footlines of the accretion curtains being fed by the accretion disk. The main (upper) accreting pole is best visible around spin phase 0 , when it points to the observer. The occurrence of the frequency $2 \omega-\Omega$ in the soft X-ray band is an indicator of two opposite, similarly bright, accretion spots, accreting via an accretion stream. This is evidence of the white dwarf's dipole axis being strongly inclined from the rotational axis, which produces similar visibility and accretion efficiency in both regions just offset by 0.5 phases in spin and beat period, respectively.

In the spectral model, we included two Gaussians to represent iron lines at 6.4 and $6.7 \mathrm{keV}$. Although they have no significant influence on the overall $\chi^{2}$, they may support the interpretation of the spin-dependent behaviour of the system. The normalisation of the $\mathrm{Fe}$-line at $6.7 \mathrm{keV}$ closely follows the shape of the normalisation of the black body component with two distinct peaks. The fluorescence line at $6.4 \mathrm{keV}$ appears to have only one peak and an eclipse-like feature at spin-phase 0.7 , which resembles the mekal component. In general, this line is interpreted to be caused by the reflection of the hard X-ray component by cold material, e.g. by the irradiated surface of the white dwarf close to the accretion region. The coupling of the normalisations of the mekal to the $6.4 \mathrm{keV}$ line supports this view in the case of MU Cam.

In the optical spectra published for MU Cam (Wei et al. 1999; Araujo-Betancor et al. 2003), there is no evience of spectral features that could be produced on the secondary star. It is therefore impossible to estimate the contribution of the late-type star to the overall flux and the distance of the system. Using orbital period-spectral type relations for cataclysmic variables determined either observationally (Smith \& Dhillon 1998) or theoretically (Beuermann et al. 1998), the companion star in MU Cam is expected to be of early M type (i.e. M1).

\section{Summary and conclusions}

We have presented the analysis of comprehensive data sets obtained simultaneously at optical, ultraviolet, soft and hard X-rays in 2005 and 2006 with the XMM-Newton observatory and the AIP $70 \mathrm{~cm}$ telescope. Our main results and conclusions may be summarized as following:

- The period tentatively derived as the white dwarf spin by Staude et al. (2003) is the dominant signal in the hard $\mathrm{X}$-ray light curves. We claim that this provides a measure of the white-dwarf rotation. By linear interpolation of the timings of the mean hard X-ray pulse maxima in the two XMM-Newton observations we measure a spin ephemeris of $B J E D\left(\max , P N_{\text {hard }}\right)=2453461.4475(8)+E \times$ $0.01374120(6)$.

- A distinct soft component in the X-ray emission, which was already visible in the ROSAT spectrum, was unambiguously detected and could be fitted well with a black body of temperature $59 \mathrm{eV}$ and $54 \mathrm{eV}$ in 2005 and 2006, respectively. MU Cam could be reliably identified as a soft IP.

- MU Cam shows changes in its accretion state between diskdominated and disk with an additional stream component during the observing interval from 2003 to 2006. These are displayed by a change in the mean optical brightness of about $1 \mathrm{mag}$, and by fundamental changes in the power spectra throughout the complete observed energy range. Such changes were also reported for other IPs (e.g. TX Col: Norton et al. 1997, FO Aqr: Beardmore et al. 1998; Evans et al. 2004), and therefore appear to be a prevalent feature of intermediate polars.

- The occurrence of the $2 \omega$ frequency in the X-ray data from 2006 and the strength of $2 \omega-\Omega$ in 2005 suggest two accretion regions at opposite positions with similar properties and visibility, at least in certain energy ranges. This may be achieved if the dipole axis of the magnetic field of the white dwarf was strongly inclined away from the rotation axis to the orbital plane.

- The strong modulation in all wavelength ranges implies a rather high inclination, whereas the absence of an X-ray (white dwarf) eclipse limits it to $\lesssim 75^{\circ}$. It is probably even lower, because no eclipse is detected in the optical, which would be a sign of a partial obscuration of the accretion disk.

- To improve our understanding of the properties of the system, future observations should include more optical photometry to refine the spin and orbital ephemerides. For studying the accretion geometry, time-resolved optical highresolution spectroscopy will be crucial, and it may help to determine an accurate orbital ephemeris. To determine the distance, near-infrared spectroscopy should be performed to be able to determine the spectral type of the secondary star and its contribution to the spectral energy distribution.

Acknowledgements. A. St. acknowledges support by the Deutsche Forschungsgemeinschaft under grant SCHW536/20-1, A.St., R.Sc., J.V., and A.N.G.M. were supported by the DLR under grant 50OR0404. We thank A. Bird, C. Knigge, and collaborators for providing the INTEGRAL spectrum of MU Cam.

\section{References}

Araujo-Betancor, S., Gänsicke, B. T., Hagen, H.-J., Rodriguez-Gil, P., \& Engels, D. 2003, A\&A, 406, 213

Barlow, E. J., Knigge, C., Bird, A. J., et al. 2006, MNRAS, 372, 224

Beardmore, A. P., Mukai, K., Norton, A. J., Osborne, J. P., \& Hellier, C. 1998, MNRAS, 297, 337

Beuermann, K., Baraffe, I., Kolb, U., \& Weichhold, M. 1998, A\&A, 339, 518

de Martino, D., Matt, G., Mukai, K., et al. 2006, A\&A, 454, 287

Evans, P. A., \& Hellier, C. 2004, MNRAS, 353, 447

Evans, P. A., Hellier, C., Ramsay, G., \& Cropper, M. 2004, MNRAS, 349, 715

Evans, P. A., Hellier, C., \& Ramsay, G. 2006, MNRAS, 369, 1229

Haberl, F., Motch, C., \& Zickgraf, F.-J. 2002, A\&A, 387, 201

Hellier, C. 2002, in The Physics of Cataclysmic Variables and Related Objects, ed. B. T. Gänsicke, K. Beuermann, \& K. Reinsch, ASP Conf. Ser., 261, 92 Hellier, C., \& Beardmore, A. P. 2002, MNRAS, 331, 407

Hellier, C., Cropper, M., \& Mason, K. O. 1991, MNRAS, 248, 233

Kazarovets, E. V., Samus, N. N., Durlevich, O. V., Kireeva, N. N., \& Pastukhova, E. N. 2006, Informational Bulletin on Variable Stars, 5721, 1

Kim, Y.-G., Andronov, I. L., Park, S.-S., et al. 2005, J. Astron. Space Sci., 22, 197

Norton, A. J., Hellier, C., Beardmore, A. P., et al. 1997, MNRAS, 289, 362

Patterson, J. 1994, PASP, 106, 209

Schwarzenberg-Czerny, A. 1989, MNRAS, 241, 153

Smith, D. A., \& Dhillon, V. S. 1998, MNRAS, 301, 767

Staude, A., Schwope, A. D., Krumpe, M., Hambaryan, V., \& Schwarz, R. 2003, A\&A, 406, 253

Staude, A., Schwope, A., Schwarz, R., Vogel, J., \& Krumpe, M. 2006, in Proceedings of the The X-ray Universe 2005, 26-30 September 2005, El Escorial, Madrid, Spain, ESA SP-604, ed. A. Wilson, 307

Suleimanov, V., Revnivtsev, M., \& Ritter, H. 2005, A\&A, 435, 191

Warner, B. 1986, MNRAS, 219, 347

Wei, J. Y., Xu, D. W., Dong, X. Y., \& Hu, J. Y. 1999, A\&AS, 139, 575

Wynn, G. A., \& King, A. R. 1992, MNRAS, 255, 83 